\title{
Wood transverse fracture analysis at the mesoscopic scale
}

\author{
L. Bigorgne ${ }^{1, a}$, M. Brunet ${ }^{1}$, and H. Maigre ${ }^{1}$ \\ ${ }^{1}$ Université de Lyon, CNRS INSA-Lyon, LaMCoS UMR5259, France
}

\begin{abstract}
The present study is an analysis of wood fracture behavior at the mesoscopic scale (i.e. the scale of growth ring). Radial TR crack growth mechanisms are complex and involve several events such as secondary cracks creation while cracks extend from one growth ring to another. Crack tip strain field of radial TR crack is measured by digital image correlation in order to better understand these phenomena. A numerical model for wood fracture has been conducted and a transverse model of spruce wood has been implemented. The simulation is realized by Material Point Method, a meshless method that discretizes material bodies into a collection of material points or particles. This promising tool improves the understanding of transverse fracture in spruce wood and can easily be used in further studies.
\end{abstract}

\section{Introduction}

Wood is a complex material, highly anisotropic and with variable mechanical properties. At the macroscopic scale $(0.1-1 \mathrm{~m})$, softwood structure is commonly described as a cylindrical orthotropic material with three main directions, the longitudinal (L), radial (R) and tangential (T) directions. At this scale, softwood is represented as a continuum material (Figure 1). For fracture mechanical characterization, the plane of the crack and the mode of crack propagation have to be specified. These crack propagation characterization are denoted with two letters related to the plane normal to the crack and the direction of crack propagation. In this study TR cracks are specifically studied. At the mesoscopic scale i.e. the scale of the annual rings (1-10mm), wood can be seen as a continuum material or as a cellular material. The cell density variation along each annual ring characterizes the material and the mechanical answer of wood. Crack propagation is more complicated and depends on annual ring data. The plane of crack growth is frequently changing while the crack extends from one annual ring to another. At the microscopic scale $(10-100 \mu \mathrm{m})$, cell shape variation and cell wall thickness lead to various fracture mechanisms [1]. In the earlywood layer, cell walls are quite thin. The main fracture mechanism is the breaking of them. In the latewood layer, thicker cell walls lead to a peeling of cell walls.

The objective of this study is to improve the analysis of radial crack growth mechanism in spruce wood transverse plane at the annual ring scale. The secondary crack apparition is in this paper specifically studied.

Cell wall damages induced by drying process are responsible of very high drying stress at the cell wall level [2]. The presented investigation is under the strong hypothesis of non significative drying damages at the mesoscopic scale.

\footnotetext{
a e-mail : loane.bigorgne@insa-lyon.fr
} 

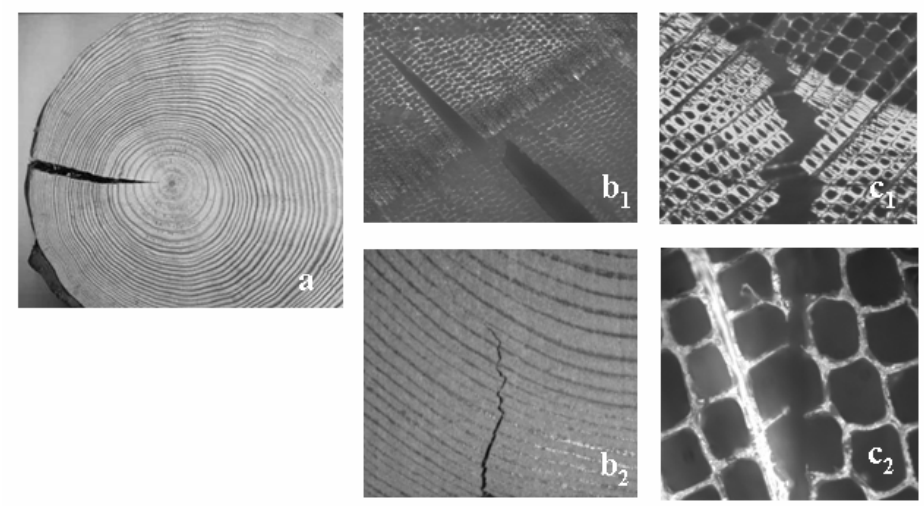

Fig. 1. Crack propagation mechanisms observation of spruce wood at several scales. At the macroscopic scale (a), radial cracks can be observed along the trunk. At the mesoscopic scale, crack path depends on crack tip position. Secondary crack creation can appear as the crack extends from one growth ring to another $\left(b_{1}\right)$. Latewood high density influences crack propagation $\left(b_{2}\right)$. At the microscopic scale, several crack mechanisms occur: peeling of cell walls in the latewood layer $\left(\mathrm{c}_{1}\right)$, cell walls breaking in the earlywood layer $\left(c_{2}\right)$.

\section{In-situ cracks analysis}

\subsection{Experimental setup}

In order to better understand fracture mechanisms at the macroscopic scale it is necessary to investigate fracture behavior at lower scales: mesoscopic scale and if necessary at the microscopic scale.

Some in-situ measurements were done under the microscope. Samples of dimension $15 * 35 * 5$ $\mathrm{mm}$ were cut respectively in the radial, tangential and longitudinal directions with a sharp fine saw. To prepare the surface for cellular study in the microscope, the surface was locally cleaned with a razor blade. Two holes were drilled for application of the load by an appropriated tensile testing setup. A crack was introduced using the fine saw and a notch was created with a new razor blade. Crack was applied in order to observe radial crack propagation to the pith of the sample. Sample was loaded under displacement control until crack propagation (Figure 2). Results and interpretations of crack path and phenomena induced by annual ring crossing are presented in the next section.

Another test has been performed in order to analyze three dimensional crack paths. Several radiographies have been done at the neutron spallation SINQ of the Paul Scherrer Institute. The imaging technique was realized with cold neutrons as it guarantees good contrast and solves some absorption issue. A sample of dimension $15 * 35 * 15 \mathrm{~mm}$ respectively in the radial, tangential and longitudinal directions has been analyzed at several step of crack propagation. A notch was introduced as previously and a hole was drilled on the TL surface in order to apply a screw into it. In this test a screw is used in order to control notch opening and crack propagation (Figure 3).

\subsection{Results and discussion}

Wood fracture behavior differs and depends on various parameters regarding to the scale of observation. At the mesoscopic scale, crack opening and propagation will depend on annual ring parameters (size, annual ring density, radial orientation ...etc.), crack parameters (orientation, direction ...etc.) and loading.

Annual ring density is an influent parameter while crack crosses a new annual ring. The specific cellular structure of spruce is responsible for this behavior. Cells density becomes more and more 
important along an annual ring. Cells present thin cell walls at the beginning of the annual ring i.e. in the earlywood area and become gradually thicker and thicker in the latewood area. The density evolution is thus quite smooth inside an annual ring whereas there is high density variation at the direct transition between latewood and new earlywood. These density variations affect fracture mechanisms. While a radial propagation occurs cracks can be stopped by the very dense latewood then a secondary radial crack appears next to the first one as one can see in Figure 2. A similar mechanical mechanism can be observed in Figure 3. While a preliminary crack reaches the dense latewood zone, it stops and a secondary crack appears next to the first one in the radial direction.
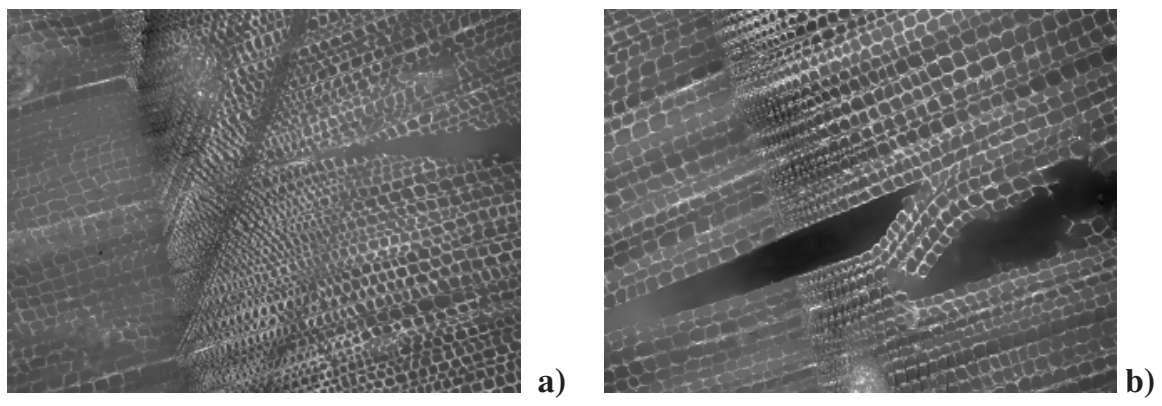

Fig. 2. In-situ microscopic crack observation while samples are loaded under displacement control. Radial crack stopped while it reaches the latewood zone (a). Secondary radial crack apparition and propagation (b)

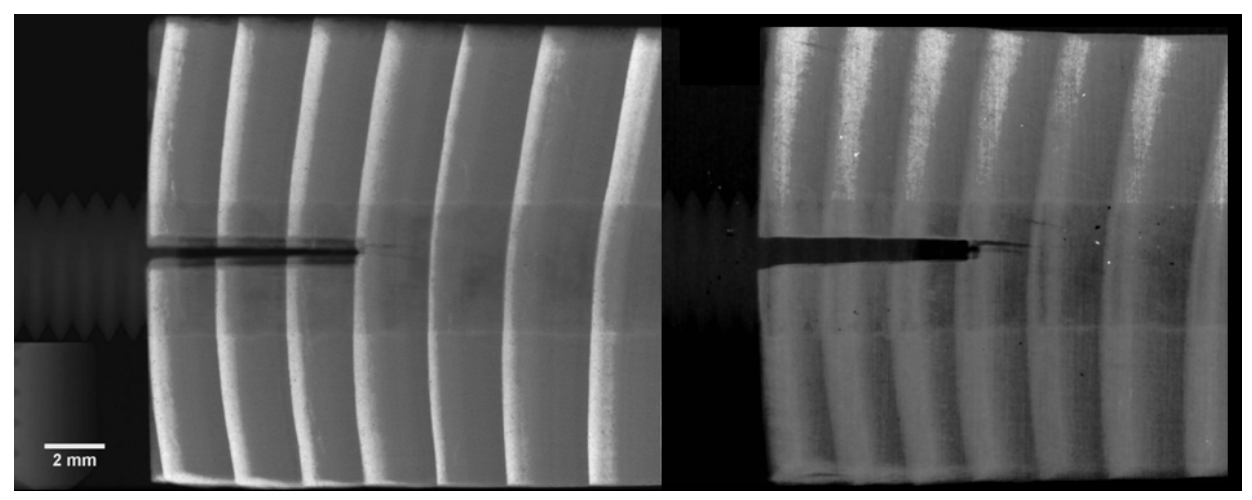

Fig. 3. In-situ radiographies of spruce wood. Observation of secondary crack apparition.

\section{Digital image correlation}

\subsection{Experimental setup}

Full field measurements realized from microscopic images may explain the origin of secondary crack. A digital image correlation analysis has been used in relation to microscopic tests.

Samples were prepared as previously. The transverse surface was sand and a carbon speckle was applied. I order to gain in measurements accuracy, wood sample as to be confined in a specific chamber with controlled temperature and humidity. This chamber has been manufactured for macroscopic tests [3]. A second chamber adapted to testing under microscope will soon run. In order to compare experimental tests with numerical simulation, tests have to be done at $25^{\circ} \mathrm{C}$ and $12 \% \mathrm{H}$ in wood. Mechanical properties of spruce wood have been calculated analytically under these conditions [3]. 
The digital image correlation setup is built from the microscope CCD-camera with imaging optic connected to a PC via a frame-grabber. The CCD camera collects images of the random pattern in the sample at several step of loading. The technique relies on that the motion of this random pattern can be detected between different steps of loading. The quality of the pattern is therefore crucial. The displacement of each pattern can be determined and so the local displacement field (Figure 4). By numerical differentiation, the strain field can be obtained at each position in the sample. The correlation technique is often a good choice to perform quality deformation measurements because of its noise tolerance and high accuracy. This was done with the software Icasoft (LaMCoS).
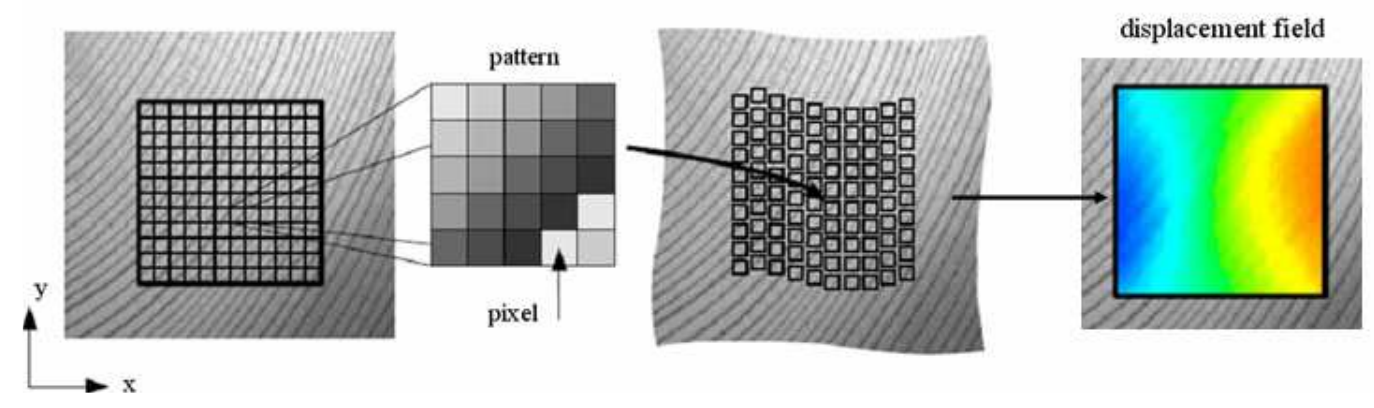

Fig. 4. Principle of the correlation algorithm. Subdivision of an image into several patterns. Pattern recognition after solicitation. Local displacements determination.

\subsection{Results and discussion}

The measurement of local strain field around the crack tip shows a specific behavior while the crack tip approaches the stiff latewood area. In-plane strain components were calculated as described previously. Tangential strain is particularly studied. One can measure high tangential strain concentration along the latewood zone while crack tip is still in the earlywood zone. Tangential strain extends in the tangential direction but is constraint in the radial direction. This may be due to the stiffness of the latewood zone. Strain map is very different from an isotropic and homogeneous material. Wood heterogeneity and anisotropy has to be considered. It is likely that secondary cracks appear at defects in the latewood layer ahead of the crack tip even when defects are located away from the primary crack. This work was also done by Thuvander in [2].

A weak area closed to the preliminary crack and associated to an increasing field concentration can lead to a secondary crack creation. It appears also that larger crack jump of crack plane are expected for wider growth ring. This phenomenon has been observed from several tests. The apparition of secondary cracks leads to radial crack deviation. Nearby cracks tend to join each other following straight lines between the different crack tips. This mechanism describes the propagation of oblique cracks.

\section{MPM numerical simulations}

In order to better understand the previous results, a numerical modeling of spruce wood is implemented using the material point method (MPM). MPM is a meshless method that discretizes material bodies into a collection of material points or particles. It was fist developed by Sulsky [4] for solving solid mechanics problems of dynamic nature. MPM combined Eulerian (provided by the grid) and the Lagrangian (provided by the material points) descriptions. The grid is fixed and used to determine spatial gradients and for solving field equations. The material points are convected by the deformation of the solid throughout the background grid and are not subjected remeshing. The 
software used in these simulations is developed by Nairn [5] and is suitable to fracture mechanics problems.

The input of these simulations is the radiographies presented in Figure 3. The real annual ring geometry is extracted and filled with adequate mechanical properties. The simulation is thus close to the real specimen morphology. Wood is implemented as a heterogeneous material as follow. Each annual ring is described as a multilayer (8 layers) composite material with gradual mechanical properties (Figure 5). Each layer is implemented as an isotropic material and a preliminary crack is added (Figure 6).
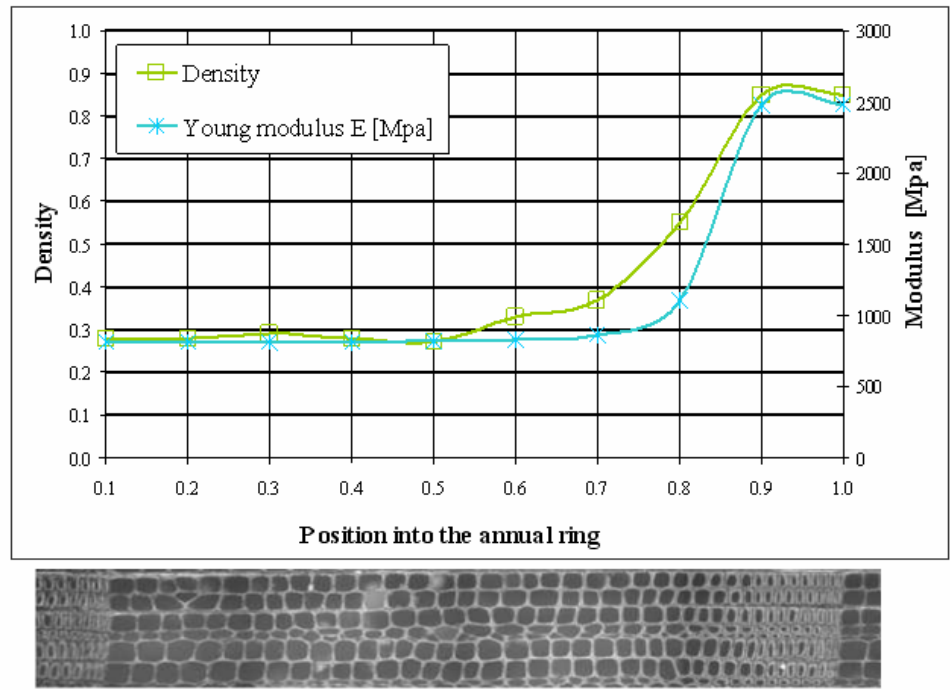

Fig. 5. Implementation of gradual isotropic mechanical properties along each annual ring

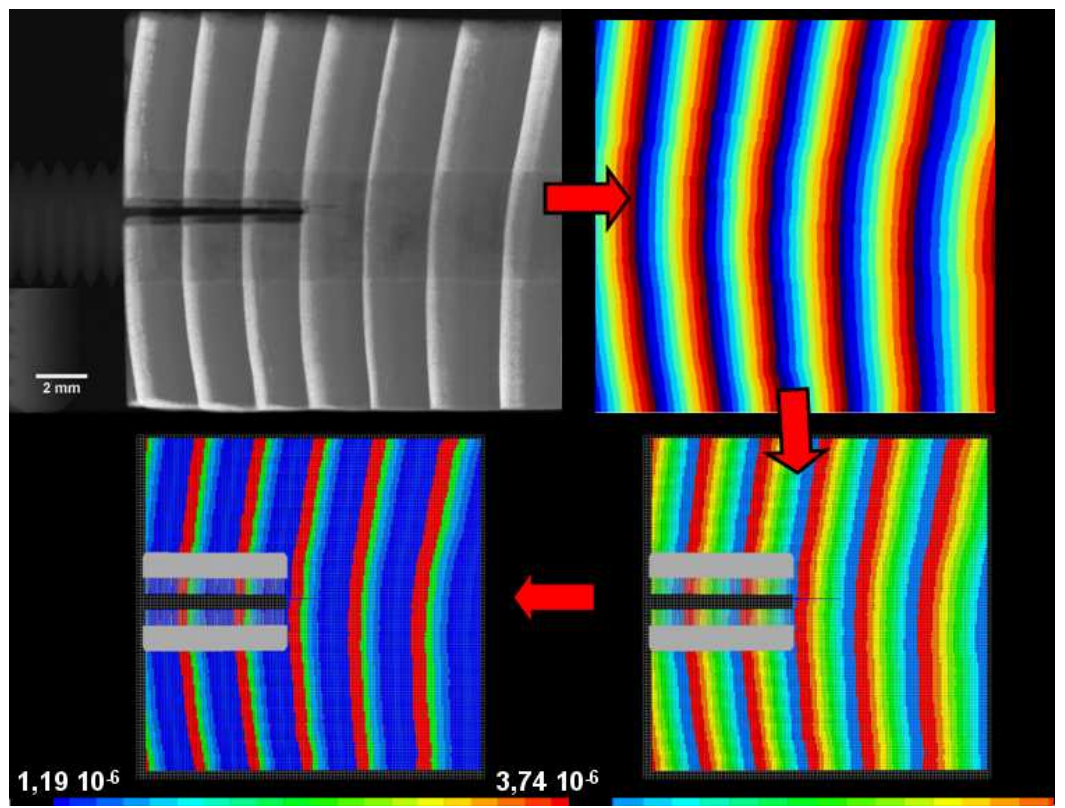

Fig. 6. Input of numerical simulation (upper left). Localization of annual rings and creation of a multilayer material (upper right). Implementation of boundary conditions and crack position. (lower right). Mass repartition in the specimen (in gram, lower left). 
It is the first step of observation. The crack tip is situated in the earlywood zone and tends to propagate in the last latewood zone. The second step of observation occurs after the secondary crack apparition and development. In these simulations, a crack is simply added and the strain field is analyzed at this stage. The numerical specimen is loaded in tension and strain field are studied (Figure 7). Tangential train field extends a lot in the tangential direction and shows high strain concentration. This is in accordance with the experimental results. Stress analysis reveals high tangential stress in the stiff latewood layer in front of the crack tip. This can be an explanation for secondary crack creation in the latewood zone. Stress and strain around the crack are strongly dependant on crack tip position along the annual ring. Stress field along a line normal to the crack ahead of the crack tip has been specifically analyzed. The results are given for seven crack tip positions along an annual ring (Figure 8). In this numerical simulation, the growth ring width is about $2.3456 \mathrm{~mm}$. The peak stress is measured and plotted with respect to the crack tip position. Crack propagation in the earlywood result in a increasing of stress (radial or tangential) as latewood is approached. This phenomenon can explain saccade crack propagation. It shows quantitatively that crack propagation is easier after latewood crossing than before it. These results are promising for further utilizations of this numerical tool.

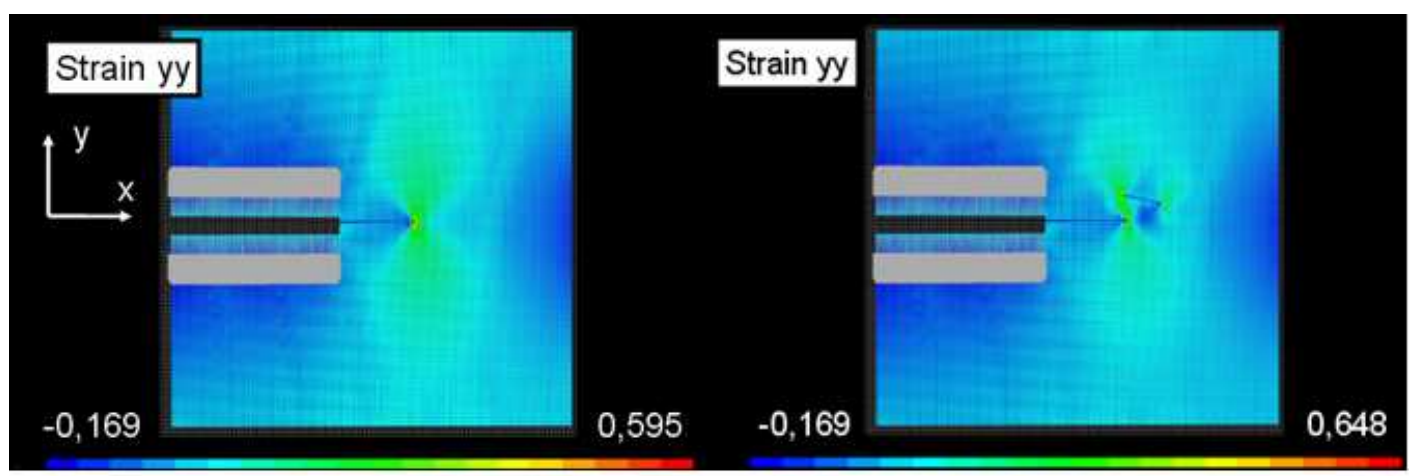

Fig. 7. Strain field analysis at several step of propagation. Single crack with crack tip in front of the latewood zone (left). Secondary crack apparition (right).

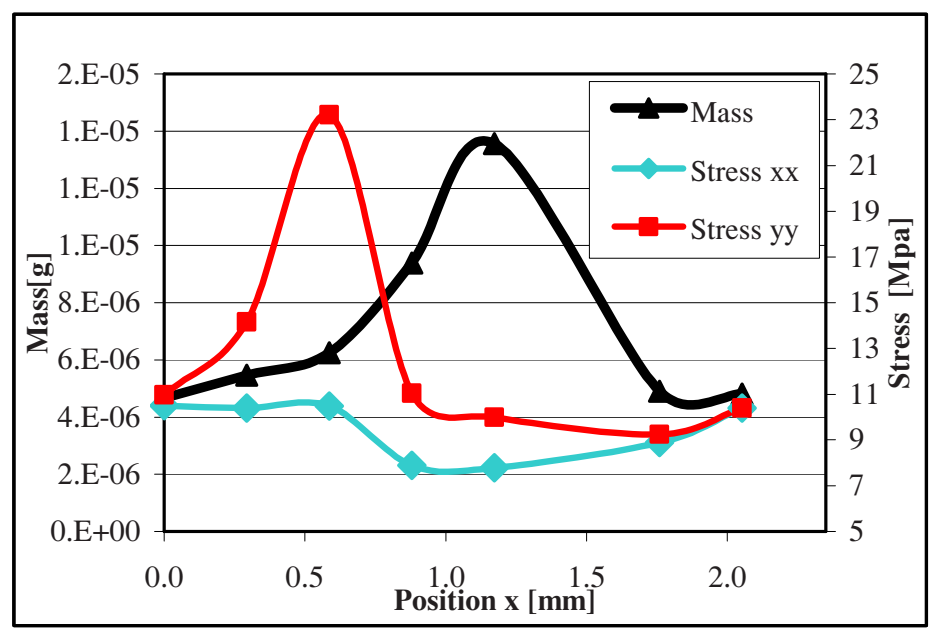

Fig. 8. Stress field ahead of the crack tip for different position of the crack along an annual ring 


\section{Conclusions}

The presented study permits experimental analysis of spruce wood fracture at the mesoscopic scale. In the earlywood zone, tangential strain field appears to extend considerably. This phenomenon was also obtained by numerical simulation. Secondary crack apparition may append in high stress concentration area in the latewood zone.

Further investigation has to be done on a more accurate determination of some crack propagation and crack direction criteria. This work is currently under study. Some crack opening measurements let suppose the preponderance of a mode I opening. Crack may be thus controlled by energetic criteria in the single mode I. Each layer i.e. each material will be affected to a specific criterion such as the value of $J_{\mathrm{Ic}}$, the critical value of the specific energy release rate. Crack direction of propagation is the one of the maximum energy release rate as measured in several simulations.

Some work has to be done too in order to better understand the secondary crack apparition. The specific energetic criteria for new crack creation have to be implemented in the code used. Crack creation may not only be due to local energetic state of the specimen and can also come from preexisting drying microcracks. This hypothesis will be further investigated in the following.

\section{References}

1. F. Thuvander, L.A. Berglund, "In situ observations of fracture mechanisms for radial cracks in wood", Journal of Material Science, 35, 6277-6283 (2000)

2. F. Thuvander, M. Sjödhal, L.A. Berglund, "Measurements of crack tip strain field in wood at the scale of growth ring", Journal of Materials Science 35, 6267-6275 (2000)

3. P. Simon, "Approche multiéchelle du comportement mécanique du bois dans le plan transverse", PhD. thesis, LaMCoS, Institut National des Sciences Appliquées de Lyon (INSA Lyon) (2009)

4. D. Sulsky, S.J. Zhou, and H.L. Schreyer, "Application of a Particle-in-Cell Method to Solid Mechanics," Computer Physics Communications, 87, 236-252 (1995)

5. J.A. Nairn, "Material Point Method Calculations with Explicit Cracks", Computer Modeling in Engineering \& Sciences, 4, 649-664 (2003) 